\title{
$\mu$-SYNTHESIS CONTROL OF FLEXIBLE MODES OF AMB ROTOR
}

\author{
Arkadiusz MYSTKOWSKI* \\ *Bialystok University of Technology, Wiejska 45 C, 15-351 Bialystok, Poland \\ a.mystkowski@pb.edu.pl
}

\begin{abstract}
In the paper the optimal robust vibration control of flexible rotor supported by the active magnetic bearings (AMBs) is investigated. The purpose of the control system is stabilization of the high speed rotor and effective control of the rotor vibration due to noncollocation, gyroscopic effects and model uncertainties. The noncollocation effect is considered and frequency modal analysis of the noncollocated AMBs system with gyroscopic effects is presented. The $\mu$-Synthesis control is applied to stabilize the rigid and flexible critical frequency modes of the rotor, with emphasis structural and parametric uncertainty. The input and output signals in AMBs system are limited by the weighting functions. The singular value analysis is used to obtain the robust performances of the closed-loop system. The stable operation and good stiffness of the high speed rotor supported magnetically is reached. The dynamical behaviour of the AMBs rotor is evaluated in the range up to $21000 \mathrm{rpm}$. The experimental tests show the effectiveness of the robust control system as well as good vibrations reduction and robustness of the designed controllers.
\end{abstract}

\section{INTRODUCTION}

The active magnetic bearings (AMBs) are used to support a rotor without mechanical contact and to vibrations control (Schweitzer et al., 1994, 2009). The AMB uses magnetic forces to levitate the rotor between two opposing electromagnetic poles. In the AMBs systems the rotor is inherently unstable. Thus, the AMBs application must be stabilized with an active feedback control system. The rotor vibrations control and vibrations compensation is a very important problem in rotating machines operating with high rotational speeds (Stephens, 1995; Knospe, 2007; Knospe et al., 1995; Mystkowski and Gosiewski, 2008b).

The dynamics of the AMBs rotor system is influenced by external disturbances (steady sinusoidal loads), nonlinearities, uncertainties and signal limits (Mystkowski and Gosiewski, 2006, 2008a, 2009). Many active vibrations control methods are used in the rotor-bearings applications, where some can be found in papers Burrows at al. (1983, 1989) and Genta (2005). The robust optimal control methods based on the $\mu$-Synthesis, $\mathrm{H}_{\infty}$ or $\mathrm{H}_{2}$ algorithms are other modern/advanced solutions (Mystkowski, 2007b; Sawicki, 2008). The $\mu$-Synthesis control permits to design the multivariable optimal robust controller for complex linear systems with any type of the uncertainties in their structure. There are many practical applications of the $\mu$-Synthesis control in the high speed rotor AMBs systems (Knospe at al., 1997; Fittro and Knospe, 1999; Fittro et al., 2003; Sawicki and Maslen, 2006). The $\mu$-Synthesis control methodology covers the practical issues like modelling of the uncertainties, selection of the optimal weighting functions and signal limits. The $\mu$-controller synthesized for the augmented plant model must meet the analysis objectives presented by the maximal singular value. Furthermore, in this method the optimized performance index has a good connection with the real AMBs system. The goal of this method is to design the stable controller which is robust to the plant dynamics variations. The $\mu$-Synthesis control procedure is natural augmentation of the $\mathrm{H}_{\infty}$ control theory with the analysis of the structural singular value (Zhou et al., 1996). Therefore, in the case of $\mu$-Synthesis control, the uncertainties are more simple to consider than in the $\mathrm{H}_{\infty}$ control. What more, the nonlinearities of the control plant can be also considered. Generally, in the robust control theory, if there are no limits, the greater robust control performances can be obtained by using greater control effort. However, the best control performances can be achieved with infinite controller gain.

The AMBs systems usually have noncollocated sensors and actuators. The noncollocation effect produces lack of interlacing of zeros and poles in the control plant model and causes a real stability problem (Preumont, 2002). Generally, the noncollocation is important in high speed rotor machines, where all the rigid and flexible frequency modes must be stable controlled (Spector and Flashner, 1989, 1990). Usually, the noncollocation provides limits in the rotor flexible modes control because of mode shape measurements faults. What more, the introduction of the interlacing repair controller is difficult especially in multiinput/multi-output (MIMO) rotor-bearings systems (Genta and Carabelli, 2000).

In the paper, the $\mu$-Synthesis control method is applied to vibrations control of the high speed AMBs flexible rotor, with emphasis noncollocation, gyroscopic effects and uncertainty. The flexible rotor supported by the AMBs is analyzed using the finite element method (FEM). The mass, stiffness and damping matrices of the flexible freefree rotor are computed and analyzed using MATLAB software. The model of the AMBs system components, which includes the magnetic bearings, rotor, power amplifiers and sensors, is presented. The influence of the noncollocated sensors and actuators on the rotor dynamics stability is evaluated. The gyroscopic effects in AMBs rotor is con- 
sidered and presented via Campbell diagram. The robust $\mu$-controller is designed due to uncertainty in the AMBs rotor model. The uncertainty is considered as variability of the electromagnetic coils inductance and rotor mode shapes perturbations. The robust control performances are verified during the experimental tests. The experimental test rig with the AMBs rotor and digital power amplifiers was designed and built. The stable high speed rotor operation and good robust performances of the closed-loop control system was reached. Finally, the success of the robust control is demonstrated through results of computer simulations and experimental results.

\section{MODEL OF AMB FLEXIBLE ROTOR SYSTEM}

To measure the rotor displacement in two radial directions, the high precision eddy-current sensors are used. The rotor displacement sensors have a high resolution and wide bandwidth up to $10 \mathrm{kHz}$, which is much over that rotor maximal angular speed. Therefore, the model of the sensors is assumed as a simple proportional gain. The rotor displacement signals are filtered by the anti-aliasing filters implemented in digital signal processor (dSpace board) in order to filter out signal components above Nyquist frequency of $4 \mathrm{kHz}$. The important consequence of poor antialiasing filtering is e.g. when the magnetic bearings react to the noise mainly in the low frequency range.

The AMBs system are operated with the current control, where at each sampling step, the digital signal processor (DSP) calculates the magnitude of the control current that is applied to the electromagnetic coils of the AMBs. These currents are generated by the digital power amplifiers based on the pulse-width-modulation (PWM). The ten channels of digital power amplifiers are used, where each of the amplifiers has an internal current control loop with a simple proportional gain controller. Thus, the coil current is measured and subtracted from the set current, which is proportional to a voltage signal of the DSP. The demanded set current is created by means of switching among a positive or negative voltage $(180 \mathrm{~V})$. The switching frequency is equal to $18 \mathrm{kHz}$. Thus, the true current oscillates around the switching frequency. For a good dynamics performances of the operating AMBs the currents should be as smooth as possible. That is why, the amplifier output lowpass filters are applied. The AMBs coils are modelled as the series interconnection of a copper resistance $R$ and an inductance $L$. The rate of the current change should be fast enough to follow the current command. However, the amplitude and frequency ranges are limited by the $R-L$ curve of the power amplifier. The model of the electromagnetic coil includes first order low-pass filter with cut-off frequency $\omega_{c}=R / L(700 \mathrm{~Hz})$ and gain of $1 \mathrm{~A} / \mathrm{V}$. The maximal value of the output current $i_{\text {max }}$ is limited to $10 \mathrm{~A}$. In the low frequency range up to the crossover frequency $\omega_{c}$, the output current is limited by the $i_{\max }$. Beyond the frequency $\omega_{c}$, the output current is limited by the coil's low-pass characteristic denoted by the $R-L$ curve. To simplify the AMBs open-loop model the dynamics of the DSP was neglected and only the gains of $\mathrm{A} / \mathrm{C}$ and $\mathrm{D} / \mathrm{C}$ converters are considered.

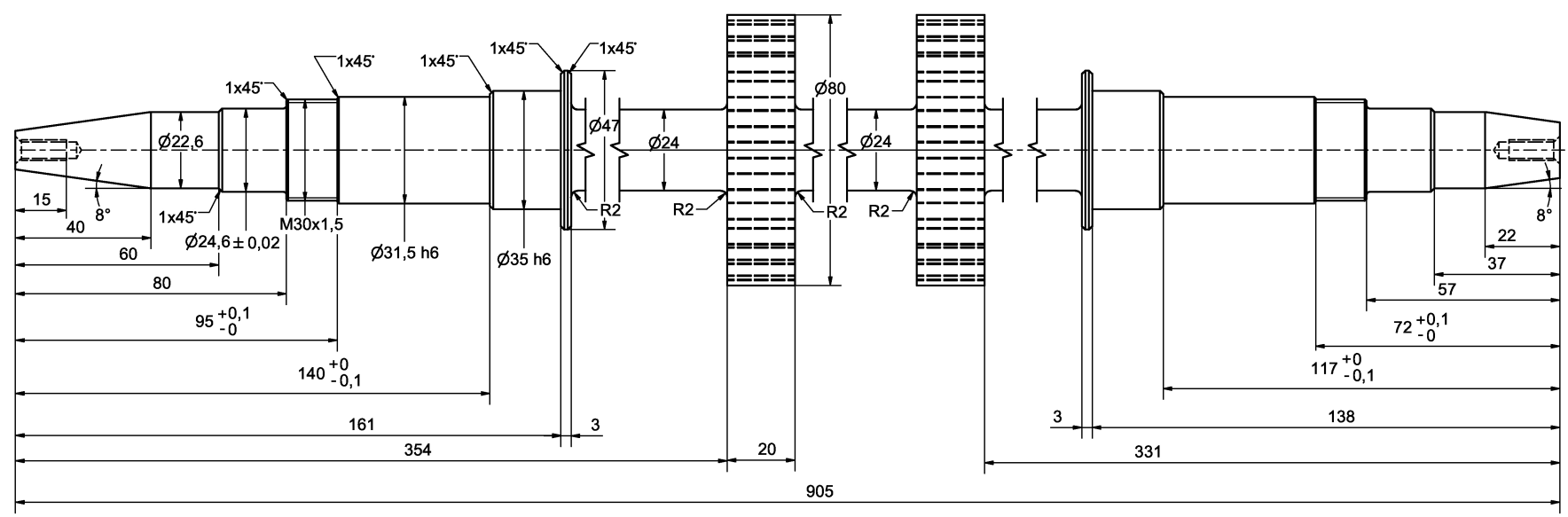

Fig. 1. Flexible rotor

The complex flexible rotor is modelled using the finite element method. Thus, the rotor was partitioned into 26 discrete elements of the simple geometry based on the Timoshenko model (Nelson, 1980). Between neighbouring elements the connecting nodes are introduced. Each of the rotor nodes has 5 degrees of freedom (DoF) and the rotor has $112 \mathrm{DoF}$. The motion equation of the free-free rotor (without external forces) is given by:

$$
M \ddot{q}+(D+\Omega G) \dot{q}+K q=0
$$

where: $M$ - symmetrical, positive definite mass matrix, $D$ - symmetrical damping matrix, $G$ - skew-symmetric gyroscopic matrix, $K$ - symmetrical, positive semi-definite stiffness matrix, $\Omega$ - rotational speed, $q$ - displacement vector.

The model of the free-free rotor is transformed to the state-space representation:

$$
\begin{aligned}
& \dot{x}=A x+B u \\
& y=C x+D u
\end{aligned}
$$


where:

$$
\begin{aligned}
A & =\left[\begin{array}{cc}
0^{q \times q} & I^{q \times q} \\
-M^{-1} K & -M^{-1}(D+\Omega G)
\end{array}\right], B=\left[\begin{array}{c}
0^{q \times 2 n} \\
M^{-1} F
\end{array}\right], \\
C & =\left[\begin{array}{ll}
S & 0^{2 l \times q}
\end{array}\right], D=\left[0^{2 l \times 2 n}\right] .
\end{aligned}
$$

The model of the external forces $F$ acting on the rotor and model of displacement sensors $S$ are given by matrices:

$$
F=\left[\begin{array}{cc}
F_{x} & 0 \\
0 & F_{y}
\end{array}\right], S=\left[\begin{array}{cc}
S_{x} & 0 \\
0 & S_{y}
\end{array}\right] .
$$

The flexible rotor supported in the AMBs is modelled by combining the model of the flexible rotor with the model of the AMBs (Losch, 2002). Thus, the model of the flexible rotor in AMBs can by described as follows:

$$
M \ddot{q}+(D+\Omega G) \dot{q}+K q=K_{s} q+K_{i} i
$$

where: $K_{s}, K_{i}$ - matrices of the AMBs displacement and current stiffness coefficients.

After transformed to modal coordinates that yields:

$$
M_{r} \ddot{q}_{r}+\left(D_{r}+\Omega G_{r}\right) \dot{q}+\left(K_{r}-K_{s r}\right) q=K_{i r} i
$$

The obtained stiffness matrix $K_{r}-K_{s r}$ is not exactly diagonal, but the diagonal elements are significantly larger than others elements. The total rotor mass is $6 \mathrm{~kg}$ and total length is $0.905 \mathrm{~m}$. The rotor dimensions are presented in Fig. 1.

\section{MODAL ANALYSIS OF AMBS GYROSCOPIC FLEXIBLE ROTOR}

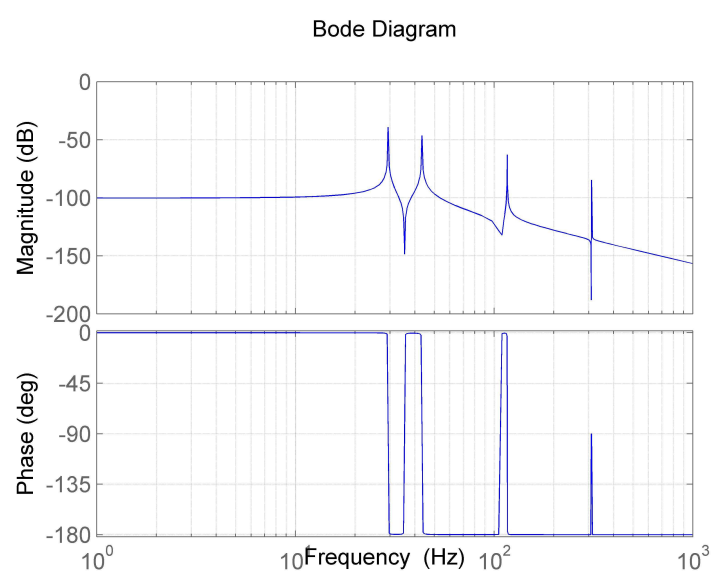

Fig. 2. Flexible rotor with $A M B s$, for $\Omega=0 \mathrm{rpm}$

The flexible rotor supported by the AMBs is analyzed using FEM such that rigid and flexible modes are analyzed. For the simulations and modal investigations the following magnetic bearings nominal parameters are considered: bias current $i_{0}=2 \mathrm{~A}$, nominal air gap $x_{0}=0.0004 \mathrm{~m}$, current stiffness $k_{i}=25 \mathrm{~A} / \mathrm{m}$ and displacement stiffness $k_{s}=97801 \mathrm{~N} / \mathrm{m}$. The modal rotor damping is chosen at $0.5 \%$ for all flexible modes. The flexible rotor model is modally reduced by using Balanced Modal Truncation Method, and only first 4 rotor modes are considered (The MathWorks, 2004).
The $5^{\text {th }}$ mode is out of operating range and has no influence. The Bode plot of the modally reduced AMBs flexible rotor is presented in Fig. 2. The two rigid modes are located at $30 \mathrm{~Hz}$ and $43 \mathrm{~Hz}$, the first flexible mode is placed at $116 \mathrm{~Hz}$.

In case of a rotating rotor (when, $\Omega>0$ ), the motion in the two planes are coupled by the gyroscopic term $\Omega G$. Thus, the gyroscopic effect causes the flexible rotor poles to move with increasing rotational frequency. The Bode plot of the AMBs flexible rotor for rotational speed $\Omega=300$ $\mathrm{Hz}$ is presented in Fig. 3. The gyroscopic effect is clearly appeared in Fig. 3 and it did not appear in Fig. 2.

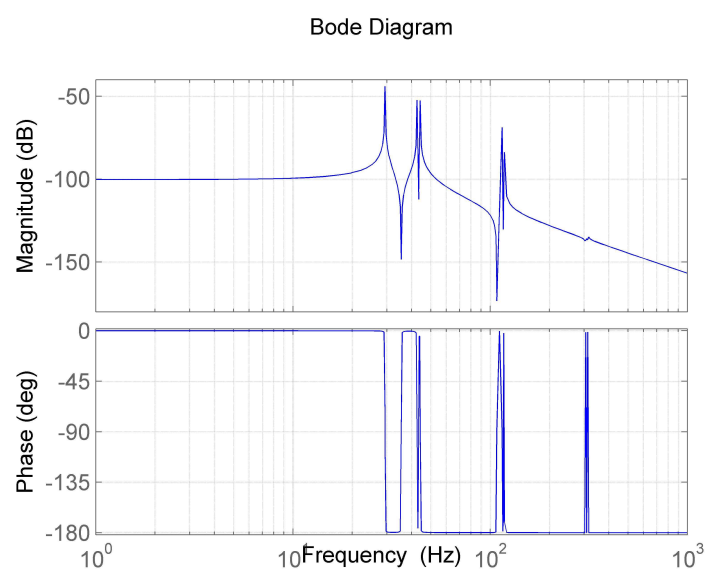

Fig. 3. Flexible rotor with $\mathrm{AMBs}$, for $\Omega=18000 \mathrm{rpm}$

\section{NONCOLLOCATINON ANALYSIS OF AMBS}

The noncollocation of eddy-current displacement sensors and magnetic actuators of the AMBs system is considered. The noncollocation effect which produces no interlaced zeros and poles of the AMBs system is a real stability problem. When a system is collocated (sensors and actuators act at the same points along the shaft), the poles and zeros are interlaced and phase is between 0 and $-180^{\circ}$ (Fig. 4). For current example, the distance between sensors and magnetic actuators is equal to $0.035 \mathrm{~m}$. Thus, AMBs system is noncollocated (Fig. 5). Here, we can notice that the poles of the AMBs model are independent of the sensor locations while the zeros are strongly sensitive to sensor locations. The poles and zeros are no longer interlaced in noncollocated case.

There is a lack of interlacing of the $5^{\text {th }}$ flexible mode (Fig. 5). They can be explained that poles no stay in the left half plane with frequency (root locus analysis) but must travel into right half plane. The noncollocated system is unstable, if the rotor mode shape node leads between sensor and actuator points, and the system is nonminimum phase. In presented example, for small no collocation, which appears above high frequency, the system is still stable in considered AMBs rotor frequency operation (phase is between 0 and $-180^{\circ}$, Fig. 5).

The interlacing repair in single-input/single-output (SISO) systems can be simply improved by adding an extra complex pair of zeros. In case of AMBs rotor system, the model is always MIMO with cross couplings between control axes. Therefore, the interlacing repair is more compli- 
cated in MIMO systems. In the paper the noncollocation effect appears at high frequency and is considered as the uncertainty model of the rotor mode shapes perturbations in the $\mu$-Synthesis control design.

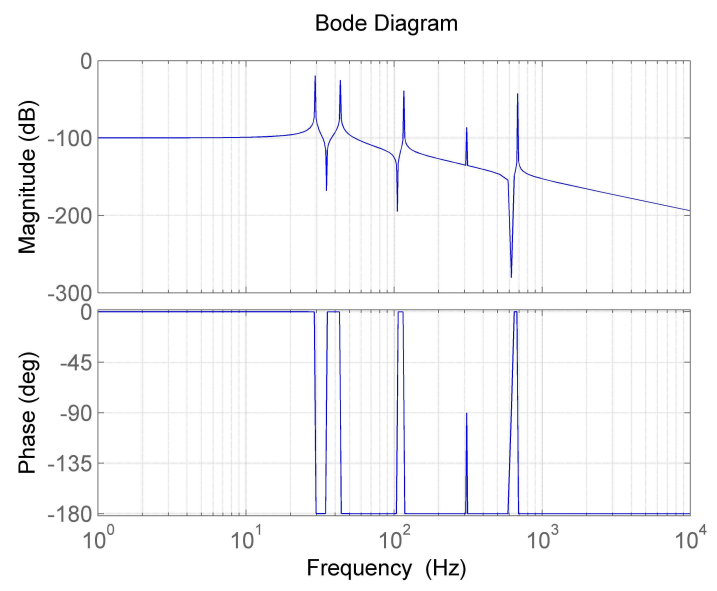

Fig. 4. Flexible rotor with AMBs for collocated sensors and actuators

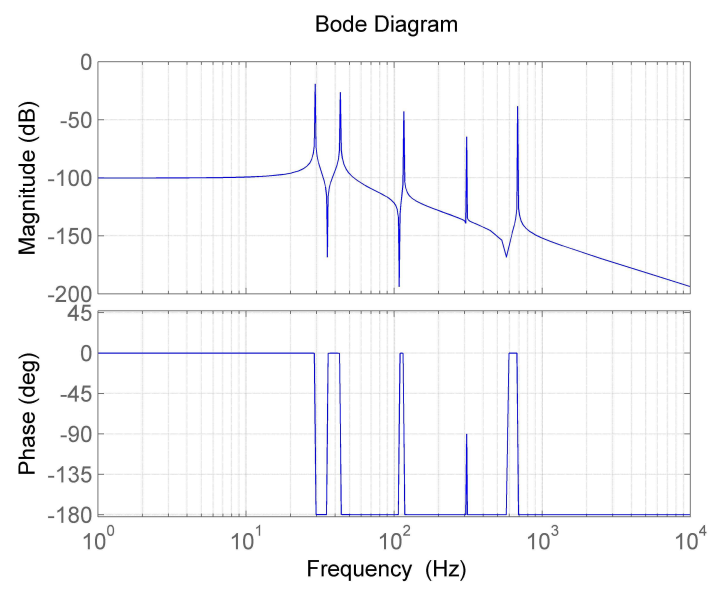

Fig. 5. Flexible rotor with AMBs for sensor located $0.035 \mathrm{~m}$ from the magnetic actuator

\section{CRITICAL SPEED ANALYSIS}

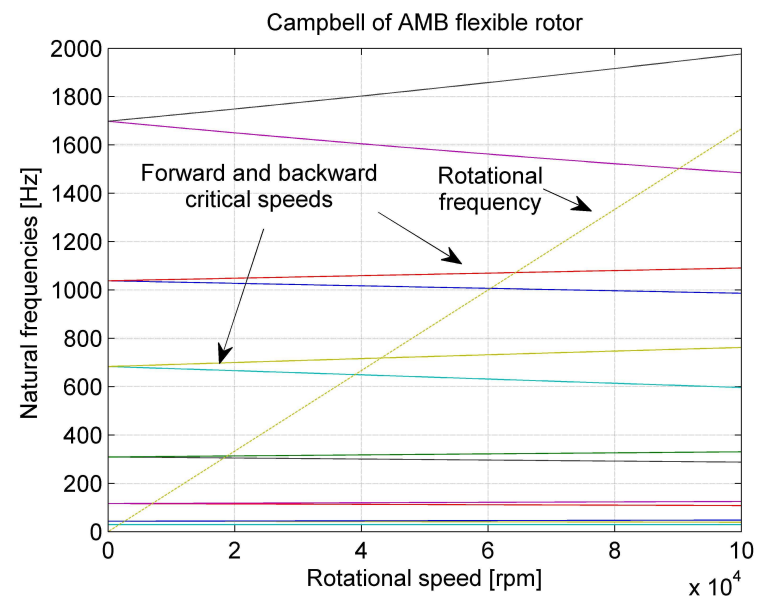

Fig. 6. Campbell diagram of the flexible rotor with AMBs for $\Omega$ from 0 to $100000 \mathrm{rpm}$

The rotor modes with increasing frequencies expose a rotation in the same direction as the rotor are called forward modes (nutations). The modes with decreasing frequencies rotate in the opposite direction are called backward modes (precessions). Fig. 6 shows the splitting of the flexible eigenfrequencies with the rotational speed from 0 to $100000 \mathrm{rpm}$. The dashed line in Fig. 6 corresponds to rotational frequency, while the solid lines correspond to backward and forward flexible mode frequencies. The crossing of flexible modes and rotational frequency called critical speeds are sensitive points of AMBs rotor application. The critical speeds have to be stable controlled during any change of the rotor speed.

\section{THE $\mu$-CONTROLLER DESIGN}

Based on the equation (5) the pole-zero distribution of the AMBs rotor open-loop model is shown in Fig. 7. Due to symmetry of the system, each pole occurs twice, once for each plane. The flexible poles are very weakly damped (damping about $0.5 \%$ ). The design process of stabilizing controllers which brings all poles to the left half plane is quite difficult. With increasing the controller gain, the poles first move to the left plane, but if the gain is further increased, they split and follow the positive and negative imaginary axis. Thus, the controller with added damping should be applied. In fact, finding process of the controller that stabilizes the all rigid modes of the flexible rotor without destabilizing the weakly damped flexible modes is difficult and complicated task.

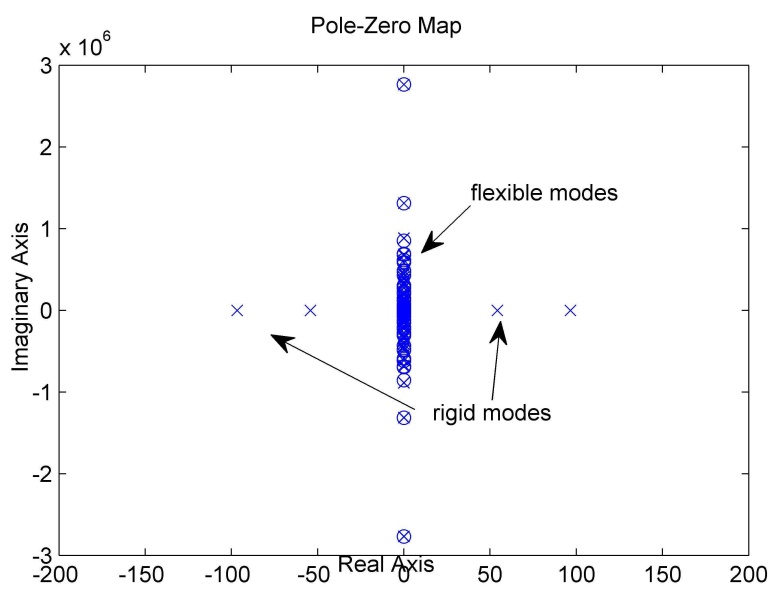

Fig. 7. Flexible rotor with AMBs (open-loop model)

In the $\mu$-Synthesis control, the cost factor is a minimal value of the norm $\|T\|_{\infty}$, where $T$ is a closed-loop transfer function. The $\mu$-Synthesis algorithm bases on the $D$ - $K$ iteration procedure is natural augmentation of the $\mathrm{H}_{\infty}$ control (Zhou et al., 1996). After performed the singular value analysis, the $\mu$-controller should satisfied the following condition in frequency domain (Zhou et al., 1996): 
$\sup _{\omega \in \mathbb{R}} \bar{\sigma}(T(j \omega)) \leq 1$

where: $\bar{\sigma}$ - maximal singular value.

Fig. 8 shows structure of $\mu$-Synthesis closed-loop system.

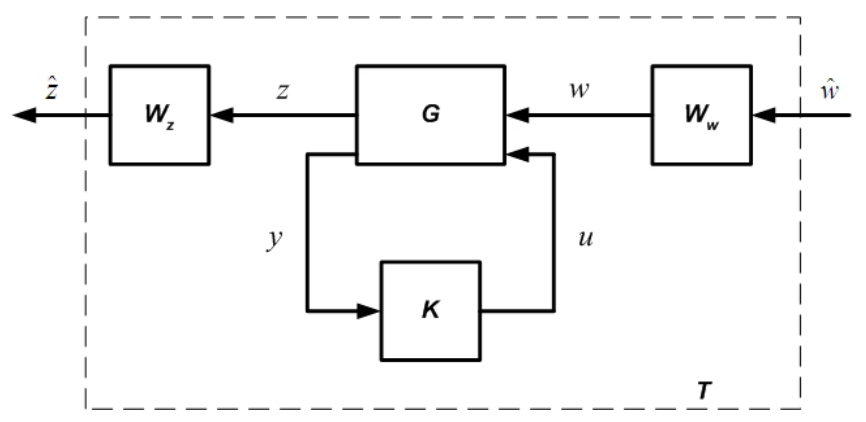

Fig. 8. $\mu$-Synthesis system, where: $G$ - augmented model of the plant, $K$ - robust controller, $W_{w}$ - weight of input signals, $W_{z}$ - weight of output signals, $z$ - any measured signals, $w$ - any disturbances (loads), $y$ - output signal, $u$ - control signal, index $\left\{{ }^{\wedge}\right\}$ means weighted signal

The robust controller is designed for augmented control plant. In this case, the augmented plant includes nominal models of: rotor, magnetic bearings, power amplifiers, sensors, DSP and also models of uncertainties and weighting functions.

The weighting functions are putted on the input and output signals like error signal, control signal and rotor displacement signal (see Fig. 9). Thus, the signals of the AMBs rotor system are scaled and the performances of the real control loop are shaped by the weighting functions (Mystkowski, 2007a). The weighting functions enable us to consider signal limits, unbalance forces and external disturbances. These functions strongly influence on the performances and robustness of the system. In robust control theory, proper chosen weighting functions must pass the following conditions (Zhou et al., 1996):

$\left\|\begin{array}{l}W_{1}(s) S(s) \\ W_{2}(s) R(s) \\ W_{3}(s) T(s)\end{array}\right\|_{\infty} \leq 1$

where: $S, R, T$ are sensitivity, control and complementary sensitivity functions.

In current example, amplitude of the weighting function $W_{3}$ putted on output signal (rotor displacements) was chosen 0.1 , because the rotor displacements should not exceed $10 \%$ of the nominal air gap (according to the vibration level at normal exploatation defined by ISO 14839-2 norm). The amplitude and bandwidth of the weighting function $W_{2}$ putted on the control signal should correspond to maximal current of the power amplifier (10 A) and maximal bandwidth $(700 \mathrm{~Hz})$. The weighting function $W_{2}$ is designed based on the $R_{0}$ control function. The proper design of the weighting function $W_{1}$ is the most important because of clear-out of steady state error, overshoot and settling time, which strongly influence on the dynamic of the AMBs closed-loop system. The $W_{1}$ weighting function are designed based on the $S_{0}$ sensitivity function. Final configu- ration of the AMBs system with weighting functions is presented in Fig. 9.

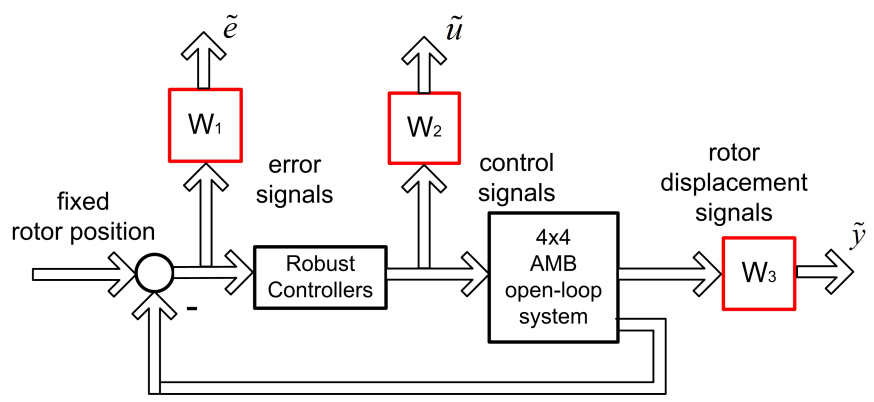

Fig. 9. AMBs weighted model

The magnetic bearing model is itself uncertain (Mystkowski and Gosiewski, 2009). The main sources of the uncertainty and nonlinearity are electromagnetic coils. The uncertainty of the AMBs system is modelled as structural uncertainty and parametric uncertainty (Knospe et al., 1996). The structural uncertainty is described by unknown norm-bounded perturbations, denoted here by $\Delta$. This perturbation acts on the nominal model $G_{N}$ via linear fractional transformation (LFT) and is represented as feedback gains connected to the control plant anywhere inside. In the rotor model, the uncertainty is connected mainly with FE nodes and can be considered as in hierarchical systems (Miatliuk et al., 2009). In this case the uncertainty was modelled as multiplicative and uncertain augmented model is given by:

$G_{R}=G_{N}\left(1+W_{m} \Delta_{m}\right)$

where: $G_{N}$ - is a nominal model, $\Delta_{\mathrm{m}}$ - perturbation satisfying $\|\Delta\|_{\infty}<1, W_{m}-$ is a stable weighting function putted on the multiplicative uncertainty.

The weighting function $W_{m}$ is given by:

$W_{m}(s)=0.1 \frac{1 / 270 s+1}{1 / 728 s+1}$

At low frequency, below $270 \mathrm{rad} / \mathrm{s}$, AMBs rotor model variation can vary up to $10 \%$ from its nominal value. After $270 \mathrm{rad} / \mathrm{s}$ the uncertainty (percentage variation) starts to increase and reaches $140 \%$ at about $728 \mathrm{rad} / \mathrm{s}$. The frequencies $270 \mathrm{rad} / \mathrm{s}$ and $728 \mathrm{rad} / \mathrm{s}$ are the values of $2^{\text {nd }}$ rigid and $1^{\text {st }}$ flexible modes of the AMBs system. Also some parameters of the AMBs system can vary from nominal values during operation. The uncertain parameters are current stiffness $k_{i}$ and displacement stiffness $k_{s}$, were the uncertainty is presented as perturbations of $\pm 5 \%$. Also, the actuator gain uncertainty is modelled as perturbations of $\pm 1 \%$. If robust controller is designed for rotational speed equal zero, there are no guarantee that the system will be stable for other values of rotational speed. The nominal value of the rotational speed $\Omega$ is equal to $10000 \mathrm{rpm}$, with uncertainty of $\pm 100 \%$. The uncertain model of the AMBs system with external signals is presented in Fig. 10.

The $\mu$-controller was computed using function provided by the Robust Control Toolbox of MATLAB called $d k s y n$ (The MathWorks, 2004). Fig. 11 shows the Bode plot of designed $\mu$-controllers computed for the AMBs system with uncertainties and weighting functions, one of them is optimized for the AMBs rotor vibrations compensation. 
The optimized $\mu$-controller was computed for the proper selected weighting functions. The magnitude of the optimized $\mu$-controller is minimalized due to maximal value of the power amplifier current. Thus, the real controller output is not saturated and good control performances are achived.

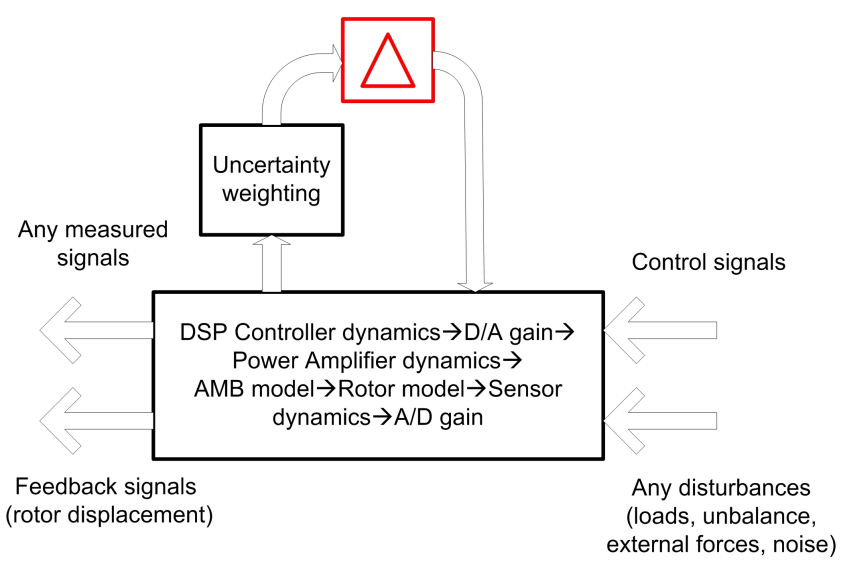

Fig. 10. Complex AMBs system

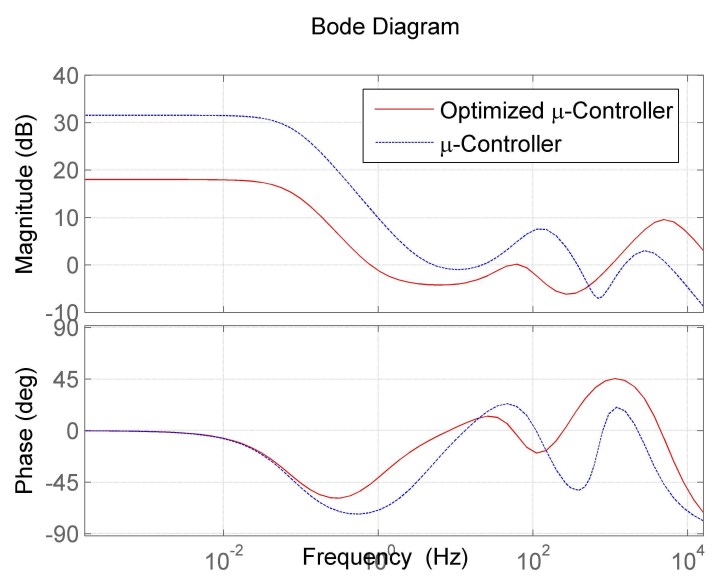

Fig. 11. Dynamics of $\mu$-controllers

\section{EXPERIMENTAL RESULTS}

The laboratory stand with the high speed AMBs rotor and digital power amplifiers was designed and built during realization of the ordered research project No PBZ-KBN109/T-10/2004 financed by the Polish Ministry of Science and Higher Education. The rotor is supported by two radial active heteropolar magnetic bearings. Each of the magnetic bearings has 8 electromagnets connected in 4 pairs in series configuration. Thus, each of the magnetic bearings demands two channels of power amplifiers. The four feedback loops are used to control the radial rotor displacements in the air gap of the radial magnetic bearings. The rotor is driven by electric motor by flexible coupling, and axial vibrations are no controlled.

The four $\mu$-controllers are used to control the rotor motion in two radial directions for two magnetic bearing planes. Thus, the four independent feedback loops with $\mu$-controllers are applied and experimentally verified. Each of the real feedback loops in the AMBs rotor application includes: rotor, heteropolar magnetic bearings, digital power amplifiers (PWM), sensors and DSP with $\mathrm{A} / \mathrm{C}$ and $\mathrm{D} / \mathrm{C}$ converters. The control algorithm is implemented as a discrete time state-space model to the DSP by using real-time interface (RTI) and real-time workshop (RTW) provided by the MATLAB. Since the $\mu$-controller cannot levitate the rotor alone, the program first brings the rotor into support under a low performance PID control algorithm. After that, the program switches to $\mu$-Synthesis control algorithm. Both, the $\mu$-Synthesis and PID control algorithms are implemented in DSP with the sampling rate of $10 \mathrm{kHz}$. The sampling rate is as high as the discrete-time controllers obtained by a bilinear approximation method good correspond to the continuous-time original system. In experimental tests the disturbances as mass unbalance, gravity loads and sensor noise are considered. The aerodynamic loads and the nonlinear phenomena like eddycurrents loses, hysteresis are neglected. Tests are performed for the rotor angular speed from 0 to $21000 \mathrm{rpm}$. The experimental set-up is presented in Fig. 12. The orbit plot of the rotor operation at $21000 \mathrm{rpm}$ is presented in Fig. 13, where because of the following rotor modes the trajectory of the geometrical centre of rotor has elliptic shape. The controlled vibrations level for the same bearings is presented in Fig. 14.

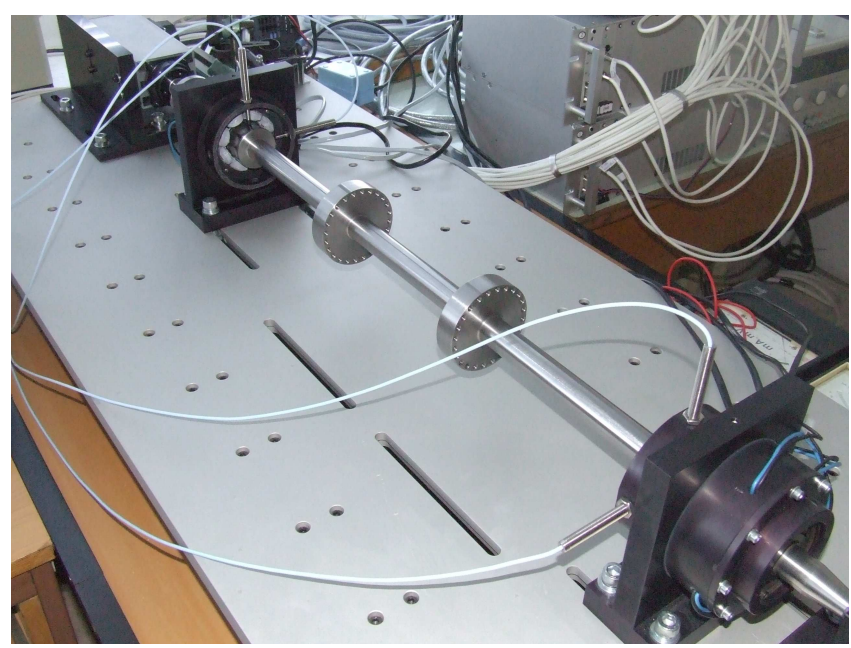

Fig. 12. Test rig

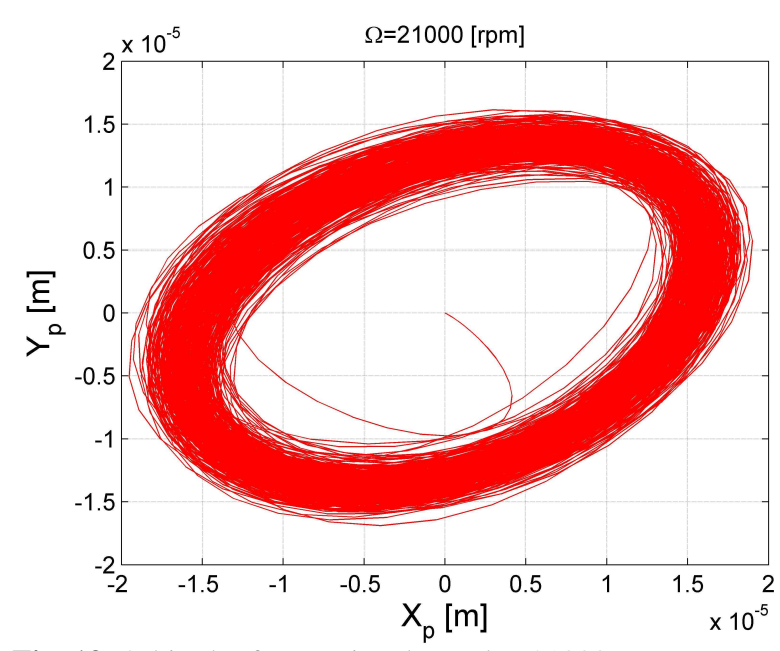

Fig. 13. Orbit plot for rotational speed at $21000 \mathrm{rpm}$ 

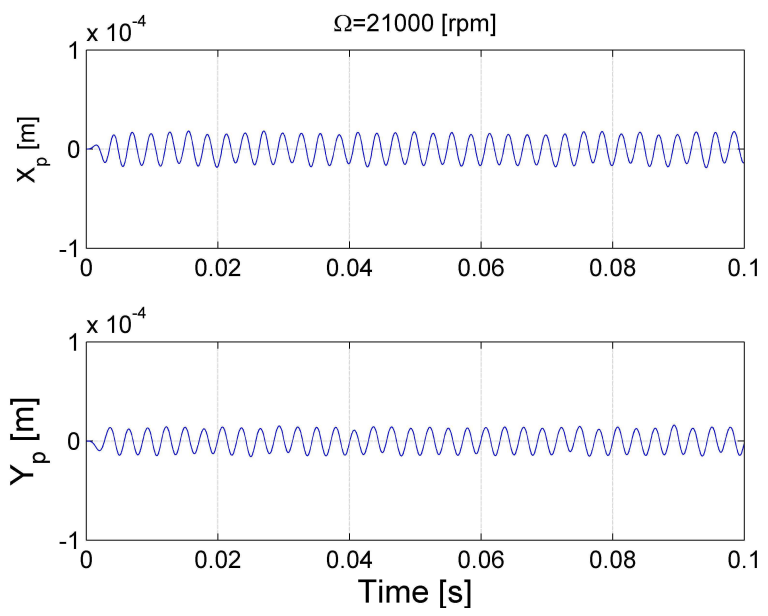

Fig. 14. Vibrations level in two radial direction for rotational speed at $21000 \mathrm{rpm}$
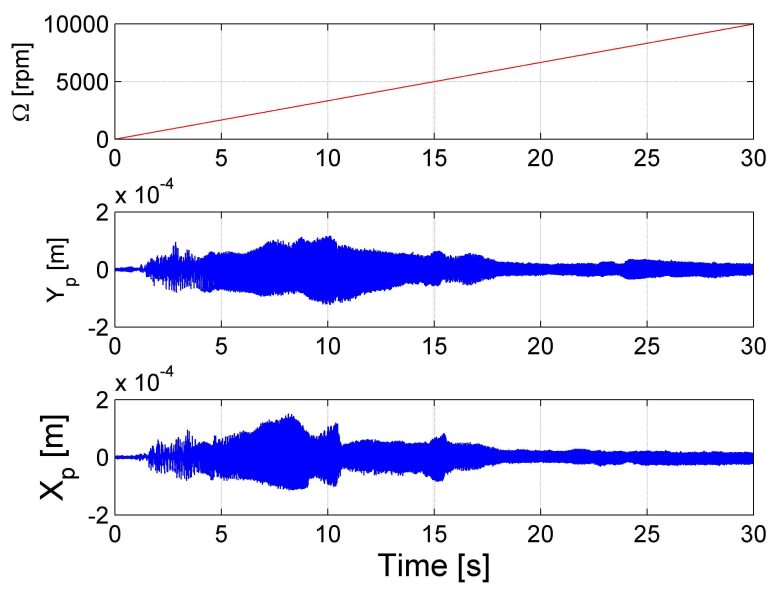

Fig. 15. Rotor acceleration test from 0 to $10000 \mathrm{rpm}$

The transient resonances (first two resonances) are shown in Fig. 15 during rotor acceleration test from 0 to $10000 \mathrm{rpm}$, where all rotor rezonanses are sucesfully controlled. The rotor speed of $21000 \mathrm{rpm}$ was not maximal of the AMBs rotor system but of the electrical drive.

\section{SUMMARY}

The paper deals with optimal robust vibrations control in wide range of the AMBs rotor speed. The presented simulations and experimental results show the potential of the $\mu$-Synthesis control applied in the AMBs rotor system. The vibrations compensation in wide range of rotation speed changes was performed successfully during experimental tests. The $\mu$-controllers have a good vibrations damping and robustness to a plant structural uncertainty. The general disadvantage of the $\mu$-Synthesis control is demanding a detailed model of the control plant. The maximal sampling frequency of the real digital processor is limited. Therefore, the order of $\mu$-controller is high and must be reduced before implementation in the real-time digital processor.

\section{REFERENCES}

1. Burrows C. R., Sahinkaya M. N, Clements S. (1989), Active Vibration Control of Flexible Rotors: an Experimental and Theoretical Study, Proceedings of the Royal Society of London, Vol. 422, 123-146.

2. Burrows C. R., Sahinkaya M. N., Clements S. (1983), Vibration Control of Multi-mode Rotor-bearing Systems, Proceedings of the Royal Society of London, Vol. 386, 77-94.

3. Fittro, R. L., Knospe, C. R. (1999), $\mu$ Control of a Highspeed Spindle Thrust Magnetic Bearing, Proceedings of the 1999 IEEE international conference on control applications, 570-575.

4. Fittro, R. L., Knospe, C. R., Stephens, L. S. (2003), $\mu$-Synthesis Applied to the Compliance Minimization of an Active Magnetic Bearing HSM Spindle's Thrust Axis, Machining Science and Tech., Vol. 7, 19-51.

5. Genta G. (2005), Dynamics of Rotating Systems, Spr.

6. Genta G., Carabelli S. (2000), Noncollocation effects on the Rigid Body Rotordynamics of Rotors on Active Magnetic Bearings, 7th Int. Symp. on Mag. B., Zurich.

7. Knospe C. R. (2007), Active Magnetic Bearings for Machining Applications, Control Engineering Practice, Vol. 15, 307-313.

8. Knospe C. R., Hope R., Fedigan S., Williams R. (1995), Experiments in the Control of Unbalance Response Using Magnetic Bearings, Mechatronics, Vol. 5, 385-400.

9. Knospe C. R., Tamer S., Fedigan S. (1996), Robustness of Adaptive Rotor Vibration Control to Structured Uncertainty, Journal of Vibration and Control, Vol. 2, 33-52.

10. Knospe, C. R., Fittro, R. L., Stephens, L. S. (1997), Control of a High Speed Machining Spindle via $\mu$-Synthesis, Proc. of 1997 IEEE international conference on control applications, 912-917.

11. Losch F. (2002), Identification and Automated Controller Design for Active Magnetic Bearing Systems, Ph.D. Dissertation, Zurich.

12. Miatliuk K, Kim Y. H., Kim K. (2009), Motion control based on the coordination method of hierarchical systems, J. of Vibroengineering, V. 11, 523-529.

13. Mystkowski A. Gosiewski Z. (2009), The Robust Control of Magnetic Suspension with Rapidly Changing of Rotor Speed, Solid State Phenomena, Vols. 147-149, 302-306.

14. Mystkowski A. (2007a), Robust Vibration Control of Rotor Supported Magnetically, Ph.D. dissertation, AGH University of Science and Technology, Cracow.

15. Mystkowski A. (2007b), Comparison of $\mathrm{H}_{\infty}$ and $\mathrm{H}_{2}$ Optimal Controllers for Active Magnetic Suspension, Developments in Machinery Design and Control, Vol. 6, 37-42.

16. Mystkowski A., Gosiewski Z. (2006), The Robust Control of Magnetic Bearings for Rotating Machinery, Solid State Phenomena, Vol. 113, 125-130.

17. Mystkowski A., Gosiewski Z. (2008a), Robust Control of Active Magnetic Suspension: Analytical and Experimental Results, Mechanical Systems \& Signal Processing, Vol. 22, No. 6, 1297-1303.

18. Mystkowski A., Gosiewski Z. (2008b), Robust Control of High Speed Rotor Supported Magnetically, 9th International Conference on Motion and Vibration Control, Munich, Germany.

19. Mystkowski A., Gosiewski Z. (2009), Uncertainty Modelling in Robust Control of Active Magnetic Suspension, Solid State Phenomena, 144, 22-26.

20. Nelson H. D. (1980), A Finite Rotating Element Using Timoshenko Beam Theory, ASME Journal of Mechanical Design, Vol. 102, 797-803. 
21. Preumont A. (2002), Vibration Control of Active Structures An Introduction 2nd Edition, Kluwer Academic Publisher, Springer.

22. Sawicki J. T. (2008), Rationale for Mu-synthesis Control of Flexible Rotor-Magnetic Bearings System, Acta Mechanica et Automatica, Vol. 2, No. 2, 67-74.

23. Sawicki J. T., Maslen E. H. (2006), AMB Controller Design for a Machining Spindle using $\mu$-Synthesis, The 10th International Symposium on Magnetic Bearings (ISMB-10), Martigny, Switzerland.

24. Schweitzer G., Bleuler H., Traxler A. (1994), Active Magnetic Bearings, Hoch. AG an der ETH, Zurich.

25. Schweitzer G., Maslen E. H, et al. (2009), Magnetic Bearings: Theory, Design and Application to Rotating Machinery, Springer.

26. Spector V. A., Flashner H. (1989), Sensitivity of Structural Models for Noncollocated Control Systems, ASME Journal of Dynamic Systems, Measurement and Control, Vol. 111, 646-655.

27. Spector V. A., Flashner H. (1990), Modelling and Design Implications of Noncollocated Control in Flexible Systems, ASME Journal of Dynamic Systems, Measurement and Control, Vol. 112, 186-193.

28. Stephens, L. S. (1995), Design and Control of Active Magnetic Bearings for a High Speed Spindle, Ph.D. dissertation, University of Virginia.

29. The MathWorks 2004, Robust Control Toolbox User's Manual, 3rd ed

30. Zhou K., Doyle J. C., Glover K. (1996), Robust and Optimal Control, Prentice-Hall, Inc.

The research work is partly financed by Bialystok University of Technology as a research own work W/WM/10/2010 conducted in the years 2010-2011. 